\title{
A Concept for Personal Wellness Management Based on Activity Monitoring
}

\author{
Elina Mattila, Ilkka Korhonen, Juho Merilahti \\ VTT Technical Research Centre of Finland \\ Tampere, Finland \\ elina.m.mattila@vtt.fi \\ Ari Nummela \\ KIHU Research Institute for Olympic Sports \\ Jyväskylä, Finland
}

\author{
Marko Myllymäki \\ IST International Security Technology \\ Helsinki, Finland \\ Heikki Rusko \\ Department of Biology of Physical Activity \\ University of Jyväskylä \\ Jyväskylä, Finland
}

\begin{abstract}
Lifestyle related health problems are increasing rapidly. Many of them could be prevented or alleviated by sufficient physical activity, enough sleep, and a balanced diet. Individual citizens have the main responsibility for their own health and wellness management in their daily lives. Motivational tools based on modern technologies can support people in this task. Vivago Personal Wellness Manager (PWM) is a concept for personal wellness management, based on continuous activity monitoring with a wrist-worn device combined with immediate feedback on daily activity, e.g., sleep and physical activity. The concept allows long-term monitoring of activity patterns and includes PC software for detailed analysis of long-term data. We studied the ability of PWM to detect different levels of activity and to estimate energy expenditure. The classification accuracy of our method was $85.4 \%$ (SD: 6.3 , range: $71.8-94.4 \%$ ). The mean absolute error of the energy expenditure estimate was $8.7 \%$ (SD: 8.0, range: $1.2-32.1 \%$ ).
\end{abstract}

Activity monitoring, energy expenditure estimation, healthenhancing physical activity, wellness management

\section{INTRODUCTION}

The cornerstones of health and wellbeing are physical activity, sleep, and healthy nutrition. The lack of or deficiency in any of these factors has serious consequences for health, for example, $35 \%$ of cardiovascular disease mortality is related to physical inactivity [1]. Sedentary lifestyles, especially time spent watching TV or sitting, have been associated with the risk of obesity and type II diabetes [2]. On the other hand, physical activity is efficient in preventing many health problems, such as high blood pressure and cholesterol, certain cancers, depression, anxiety, and even mortality [1]. Sleep, both insufficient and excessive, increases the risk of cardiovascular diseases, diabetes, mental problems, accidents, and all-cause mortality [3], [4].

The prevalence of poor lifestyles is increasing rapidly. About two thirds of Europeans are not sufficiently physically active [5]. One third of Finnish adults suffer from symptoms of insomnia, and the prevalence increases with age to more than $50 \%$ in the over 65 -year-olds [6]. Lifestyle related health risks and diseases cause high costs to society, both as direct healthcare costs and indirect costs due to lost productivity and work ability, sickness absences, and early retirement. In the UK, the estimated annual costs of physical inactivity are between 3 and 12 billion euros [7].

Health-enhancing physical activity is defined as "any form of physical activity that benefits health and functional capacity without undue harm or risk" [8]. Modern recommendations incorporate moderate intensity activity and everyday informal physical activity alongside formal exercise. The American College of Sports Medicine and the American Heart Association recommend 30 minutes of moderate intensity physical activity on at least five days a week; or 2-3 hours of formal exercise per week; or a combination of the two. Daily moderate intensity activity can be accumulated in 10-minute sessions [9], [10].

Due to the scale of the problem, the society does not have resources to provide interventions for and individually support people with sub-optimal health behavior. Thus, people need to take more responsibility for their own health and wellness. However, motivating people to work for their health is not easy in the modern, busy world with sedentary working life.

IST Vivago (IST International Security Technology, Helsinki, Finland) is a wrist-worn activity monitor, which has a movement sensor capable of measuring the muscle movements inside the wrist in addition to major hand and body movements [11]. A new model of IST Vivago, Personal Wellness Manager (PWM), has been developed, featuring improved measurement technology; a user interface and feedback in the wrist device; and a watch [12]. PWM measures continuous activity and gives feedback on sleep, physical activity, and energy expenditure. The PWM concept includes PC software for detailed analysis and long-term feedback. In this paper, we present the concept for personal wellness management based on PWM. The objective of this paper is to develop and test a method for estimating physical activity related measures with PWM.

\section{Personal Wellness Manager ConcePt}

The previous version of IST Vivago [11] is an intelligent social alarm system for the elderly, consisting of a wrist device 
with measurement sensors and an alarm button. Activity monitoring from the wrist enables detecting abnormal activity states and generating automatic alarms. Vivago activity signal has been found to provide information on circadian patterns, which differ between healthy and demented elderly people [13], and correlate with changes in health status [14]. The activity measurement of Vivago is comparable to actigraphy in sleep/wake studies and suitable for long-term monitoring of sleep/wake patterns [15]. Vivago also provides information on the depth of sleep [16].

The Personal Wellness Manager concept, based on a new model of IST Vivago, is designed to support the wellness management of working age people (Figure 1). PWM has improved measurement technology, which has enabled the development of new physical activity related features. The wrist device includes a watch and a user interface, which enables providing immediate feedback to the user. PWM monitors the activity of the user continuously and uses this information to analyze the activity patterns of the user. The device classifies activity into sleep, passivity, light activity, and health-enhancing activity and calculates the times spent in each activity state. PWM also estimates daily energy expenditure. Feedback on these parameters is displayed on the watch screen as simple graphs and parameters. These algorithms run in the PWM device. The device also stores up to four weeks worth of continuous activity data, which can be wirelessly transferred to a PC using a USB (Universal Serial Bus) radio frequency interface. PC analysis software enables detailed analysis of the data, richer feedback, and long-term follow-up of activity parameters.
Wrist is an unobtrusive location for measurement and can be used for long-term monitoring, even up to years. PWM monitors two of the three important factors of health, physical activity and sleep, providing a more comprehensive view on the health-related behavior of the user. As the device contains a watch, it has also other important functionality for the user in addition to wellness management, increasing the motivation to wear it continuously. Feedback on the device's screen helps the user to assess his/her behavior by providing clear feedback, e.g., daily duration of sleep or health-enhancing activities, which can be directly compared to recommendations. The device also acts as a constant reminder of wellness management, and may motivate the user to take better care of him/herself. This concept may be used independently by a citizen in his/her personal health management or as a part of a health promotion intervention.

\section{PHYSICAL ACTIVITY FEATURES}

\section{A. Materials and Methods}

Twenty volunteer subjects (10/10 men/women) participated in laboratory measurements at KIHU Research Institute for Olympic Sports, Jyväskylä, Finland. The inclusion criteria were: age between 18 to 40 years at the time of measurement and body mass index (BMI) between 18.5 and $30.0 \mathrm{~kg} / \mathrm{m}^{2}$ (normal weight or overweight, no obesity). Exclusion criteria were pregnancy and any disease or injury preventing physical activity. The background information of the subjects is presented in Table I. The study was approved by the ethical committee of the University of Jyväskylä.
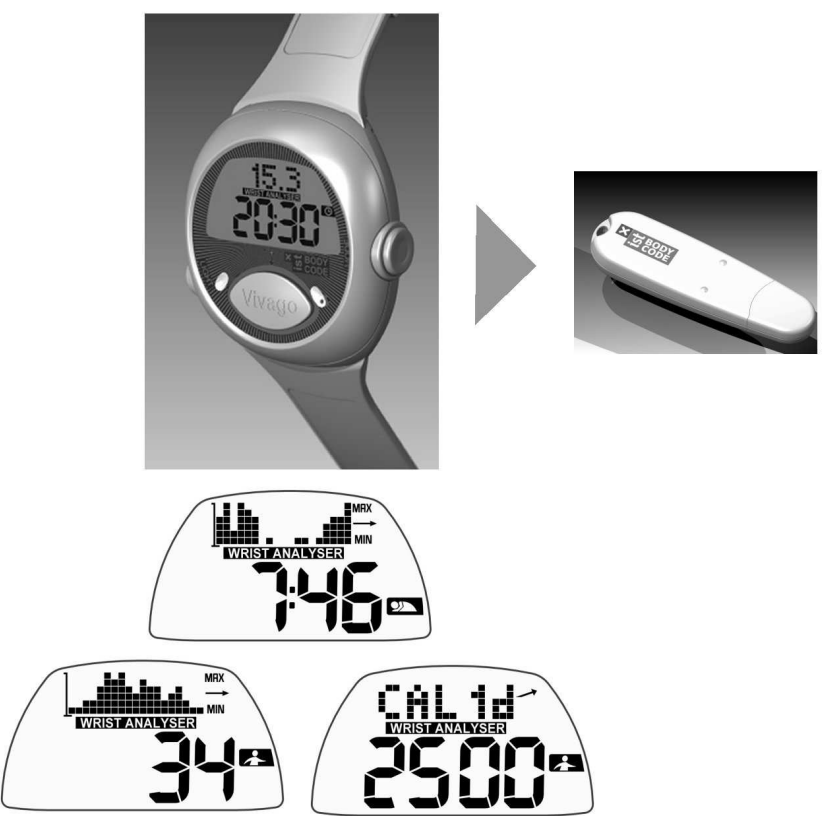

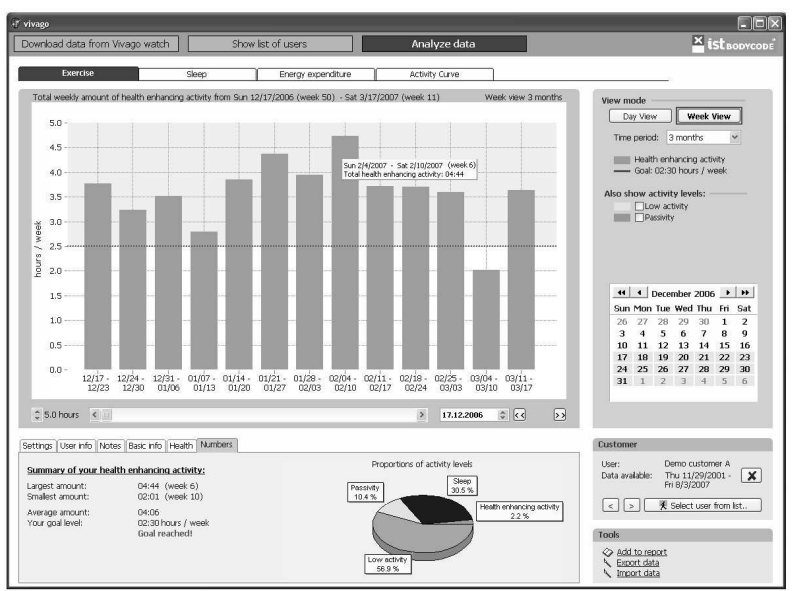

Figure 1. Vivago Personal Wellness Manager concept including the PWM device, USB radio frequency interface, and analysis software. 
TABLE I. STUDY SUBJECTS' BACKGROUND INFORMATION

\begin{tabular}{|c|c|c|c|}
\hline & $\begin{array}{c}\text { Women }(N=10) \\
\text { mean } \\
(S D ; \text { min-max })\end{array}$ & $\begin{array}{c}\text { Men }(N=10) \\
\text { mean } \\
(S D ; \text { min-max })\end{array}$ & $\begin{array}{c}\text { All }(N=20) \\
\text { mean } \\
(S D ; \text { min-max })\end{array}$ \\
\hline $\begin{array}{c}\text { Age } \\
\text { [years] }\end{array}$ & $\begin{array}{c}28.1 \\
(4.3 ; 22-37)\end{array}$ & $\begin{array}{c}28.8 \\
(3.5 ; 26-36)\end{array}$ & $\begin{array}{c}28.5 \\
(3.8 ; 22-37)\end{array}$ \\
\hline $\begin{array}{l}\text { Height } \\
{[\mathrm{cm}]}\end{array}$ & $\begin{array}{c}165 \\
(4.0 ; 158-171)\end{array}$ & $\begin{array}{c}178 \\
(5.1 ; 171-184)\end{array}$ & $\begin{array}{c}171 \\
(8.0 ; 158-184)\end{array}$ \\
\hline $\begin{array}{c}\text { Body } \\
\text { mass }[\mathrm{kg}]\end{array}$ & $\begin{array}{c}62.5 \\
(5.0 ; 54.7-69.5)\end{array}$ & $\begin{array}{c}74.0 \\
(10.0 ; 64.3-100.3)\end{array}$ & $\begin{array}{c}70.3 \\
(11.1 ; 54.7-100.3)\end{array}$ \\
\hline $\begin{array}{c}\text { BMI } \\
{[\mathrm{kg} / \mathrm{m} 2]}\end{array}$ & $\begin{array}{c}23.1 \\
(1.8 ; 20.8-26.5)\end{array}$ & $\begin{array}{c}24,7 \\
(2.4 ; 21.2-30.0)\end{array}$ & $\begin{array}{c}23.9 \\
(2.2 ; 20.8-30.0)\end{array}$ \\
\hline $\begin{array}{l}\text { Physical } \\
\text { activity }^{\mathrm{a}}\end{array}$ & $\begin{array}{c}5.7 \\
(1.4 ; 3-7)\end{array}$ & $\begin{array}{c}5.1 \\
(1.4 ; 3-7)\end{array}$ & $\begin{array}{c}5.4 \\
(1.4 ; 3-7)\end{array}$ \\
\hline
\end{tabular}

The duration of each measurement was about one hour and the protocol consisted of twenty-seven tasks including rest, light and moderate intensity everyday activities, and light to strenuous exercises (Table II).

True energy expenditure was measured by a portable indirect calorimeter, Oxycon Mobile (Jaeger, Hoechberg, Germany). Wrist activity was measured from the non-dominant wrist by a PWM. Beat-to-beat heart rate was measured by Suunto t6 heart rate monitor (Suunto, Vantaa, Finland) and Alive Heart Monitor (Alive Technologies Pty, Ltd., Arundel, Queensland, Australia). A measurement log of the true start and end times of each task was kept.

The measurement log was used to synchronize data from different devices and segment them into tasks. In order to obtain reliable steady-state data from each task, samples from the beginnings and ends of tasks were removed. The amount of data removed was determined by visual inspection and ranged from 10 to 60 seconds in the beginnings and 5 to 20 seconds in the ends of the tasks. Furthermore, tasks that were preceded by high physical load were removed, because there were no pauses between the tasks and thus some of the energy expenditure of strenuous tasks was summed to the following low intensity tasks by the reference method (indirect calorimeter). The removed tasks were: 16, 18, 20, 21, and 27. The average total duration of the data selected for analysis was $34.9 \mathrm{~min}$ (SD: 0.589 , range: $33.4-35.7 \mathrm{~min}$ ). The analysis was performed in segments of 30 seconds. Heart rate based estimates of energy expenditure were determined from beat-tobeat heart rate data using Firstbeat Pro software (version 1.4.1.5, Firstbeat Technologies Ltd., Jyväskylä, Finland) [18].

A model to classify the activities into passivity, light and high activity was developed. The reference classes are presented in Table II. A model for estimating energy expenditure was developed. Classification and energy expenditure estimation were tested using leave-one-out cross validation.

\section{B. Results}

The classification accuracy for the three classes, averaged over all subjects, was $85.4 \%$ (SD: 6.3, range: 71.8-94.4\%).
TABLE II. MEASUREMENT PROTOCOL TASKS. REMOVED TASKS ARE IN ITALICS. REFERENCE CLASSES: P = PASSIVITY, L = LIGHT ACTIVITY, $\mathrm{H}=\mathrm{HIGH}$ ACTIVITY

\begin{tabular}{|c|c|c|c|}
\hline \multirow[b]{2}{*}{$\begin{array}{c}\text { Task } \\
\text { number }\end{array}$} & \multicolumn{3}{|c|}{ Measurement protocol } \\
\hline & Task description & $\begin{array}{c}\text { Durati } \\
\text { on } \\
\text { [min] }\end{array}$ & $\begin{array}{l}\text { Ref. } \\
\text { Class }\end{array}$ \\
\hline 1 & Rest sitting & 1 & $\mathrm{P}$ \\
\hline 2 & Rest lying down & 1 & $\mathrm{P}$ \\
\hline 3 & Rest sitting & 1 & $\mathrm{P}$ \\
\hline 4 & Rest standing & 1 & $\mathrm{P}$ \\
\hline 5 & Rest lying down & 1 & $\mathrm{P}$ \\
\hline 6 & Rest standing & 1 & $\mathrm{P}$ \\
\hline 7 & Rest sitting, hands on the table & 2 & $\mathrm{P}$ \\
\hline 8 & $\begin{array}{l}\text { Reading a paper, sitting (non-dominant } \\
\text { hand turns pages) }\end{array}$ & 2 & $\mathrm{~L}$ \\
\hline 9 & Typing on a computer & 2 & $\mathrm{~L}$ \\
\hline 10 & $\begin{array}{c}\text { Walking on flat surface, constant } \\
\text { distance }\end{array}$ & 3 & $\mathrm{H}$ \\
\hline 11 & $\begin{array}{l}\text { Walking with a burden, constant } \\
\text { distance }\end{array}$ & 3 & $\mathrm{H}$ \\
\hline 12 & $\begin{array}{l}\text { Moving objects, sitting (non-dominant } \\
\text { hand used) }\end{array}$ & 3 & $\mathrm{~L}$ \\
\hline 13 & Vacuuming & 3 & $\mathrm{~L}$ \\
\hline 14 & Moving objects, walking & 3 & $\mathrm{~L}$ \\
\hline 15 & Bicycle ergometer, $50 \mathrm{~W}, 60 \mathrm{rpm}$ & 3 & $\mathrm{~L}$ \\
\hline 16 & $\begin{array}{c}\text { Walking, moving from one task to } \\
\text { another }\end{array}$ & 1 & - \\
\hline 17 & Walking up stairs & 1 & $\mathrm{H}$ \\
\hline 18 & Walking down stairs & 1 & - \\
\hline 19 & Walking up stairs with a burden & 1 & $\mathrm{H}$ \\
\hline 20 & Walking down stairs with a burden & 1 & - \\
\hline 21 & $\begin{array}{c}\text { Walking, moving from one task to } \\
\text { another }\end{array}$ & 1,5 & - \\
\hline 22 & Walking on a treadmill $5 \mathrm{~km} / \mathrm{h}$ & 4 & $\mathrm{H}$ \\
\hline 23 & Walking on a treadmill $6 \mathrm{~km} / \mathrm{h}$ & 4 & $\mathrm{H}$ \\
\hline 24 & Walking on a treadmill $7 \mathrm{~km} / \mathrm{h}$ & 4 & $\mathrm{H}$ \\
\hline 25 & Running on a treadmill $7 \mathrm{~km} / \mathrm{h}$ & 4 & $\mathrm{H}$ \\
\hline 26 & Running on a treadmill $8 \mathrm{~km} / \mathrm{h}$ & 4 & $\mathrm{H}$ \\
\hline 27 & Rest sitting, hands on the table & 2 & - \\
\hline
\end{tabular}

The mean absolute error of PWM based estimate of total energy expenditure was $8.7 \%$ and of the heart rate based estimate $20.1 \%$ (Table III).

The correlation between the true energy expenditure, measured with indirect calorimeter, and the PWM estimate was 0.75 (SD: 0.13, range: 0.30-0.88). The correlation between the true expenditure and the heart rate based estimate was 0.93 (SD: 0.08, range: 0.60-0.98). 
TABLE III. MEASURED ENERGY EXPENDITURE AND PWM AND HEART RATE ESTIMATES AND THEIR ERRORS. "F”' IN SUBJECT IDENTIFICATION STANDS FOR FEMALE, "M" FOR MALE SUBJECT.

\begin{tabular}{|c|c|c|c|c|c|}
\hline Subject & $\begin{array}{c}\text { Measured } \\
{[\text { [kcal }]}\end{array}$ & $\begin{array}{c}P W M \\
\text { estimate } \\
{[\text { kcal }]}\end{array}$ & $\begin{array}{c}\text { Heart rate } \\
\text { estimate } \\
\text { [kcal] }\end{array}$ & $\begin{array}{c}P W M \\
\text { error } \\
{[\%]}\end{array}$ & $\begin{array}{c}\text { Heart rate } \\
\text { error }[\%]\end{array}$ \\
\hline $\mathrm{F} 2$ & 183 & 193 & 99 & 5.6 & 45.6 \\
\hline F3 & 167 & 156 & 164 & 6.5 & 1.8 \\
\hline $\mathrm{F} 4$ & 170 & 160 & 138 & 6.0 & 19.1 \\
\hline F6 & 188 & 196 & 187 & 4.6 & 0.6 \\
\hline F7 & 166 & 159 & 137 & 4.1 & 17.3 \\
\hline F8 & 187 & 190 & 144 & 1.8 & 22.8 \\
\hline F9 & 170 & 225 & 226 & 32.1 & 33.1 \\
\hline F10 & 165 & 142 & 116 & 14.1 & 29.6 \\
\hline F11 & 165 & 123 & 120 & 25.4 & 26.8 \\
\hline F12 & 146 & 158 & 154 & 7.7 & 5.4 \\
\hline M1 & 192 & 210 & 169 & 9.7 & 12.0 \\
\hline M2 & 206 & 215 & 189 & 4.3 & 8.0 \\
\hline M3 & 205 & 223 & 127 & 9.1 & 38.0 \\
\hline M4 & 182 & 189 & 144 & 3.7 & 20.8 \\
\hline M5 & 216 & 221 & 183 & 2.4 & 15.4 \\
\hline M6 & 184 & 216 & 159 & 17.1 & 13.8 \\
\hline M7 & 184 & 170 & 116 & 7.5 & 36.7 \\
\hline M8 & 198 & 214 & 166 & 7.8 & 16.4 \\
\hline M9 & 220 & 215 & 155 & 2.3 & 29.6 \\
\hline M10 & 271 & 274 & 248 & 1.2 & 8.5 \\
\hline Mean & 188 & 192 & 157 & 8.7 & 20.1 \\
\hline SD & 27 & 36 & 37 & 8.0 & 12.6 \\
\hline Min & 146 & 123 & 99 & 1.2 & 0.6 \\
\hline Max & 271 & 274 & 248 & 32.1 & 45.6 \\
\hline
\end{tabular}

Fig. 2 presents the measured energy expenditure and the estimates based on PWM data and heart rate in different tasks as kilocalories per minute, averaged over all subjects. The PWM method was the most accurate in estimating task 2 (rest lying down), 13 (vacuuming), 17 (walking up stairs), and 22 and 23 (walking on a treadmill at 5 and $6 \mathrm{~km} / \mathrm{h}$ ). The method overestimated energy expenditure in most resting tasks, tasks 8 (reading sitting), 9 (typing on a computer), 10 and 11 (walking with and without a burden), and 12 (moving objects sitting). Energy expenditure was underestimated in tasks 14 (moving objects walking), 15 (bicycle ergometer), 19 (walking up stairs with a burden), and 24 to 26 (walking and running on a treadmill at 7 and $8 \mathrm{~km} / \mathrm{h}$ ). The heart rate based method was the most accurate in tasks 12 (moving objects sitting), 17 (walking up the stairs), and 25 and 26 (running on a treadmill at 7 and 8 $\mathrm{km} / \mathrm{h}$ ). The method slightly overestimated some of the tasks in

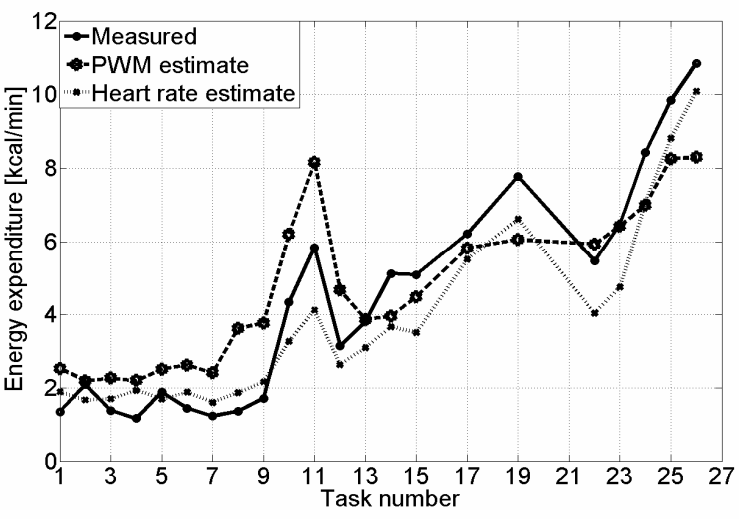

Figure 2. Energy expenditure in different tasks averaged over all subjects.

the beginning of the measurement (tasks 1, 3, 4, and 6-9) and underestimated energy expenditure in the rest of the tasks.

\section{DISCUSSION}

The new PWM concept offers a comprehensive view on 24-hour activity, including quantification of both healthenhancing activity and sedentary activities, and sleep. The sleep detection accuracy has been earlier reported to be similar to actigraphy [15]. In this study, we focused on activity classification and energy estimation performance.

The activities were classified in PWM as passivity, light activity, and high activity. The classification performance of the method was $85.4 \%$, which is good considering that only wrist activity measures were used for classifying the activities. Since the classification was done in segments of 30 seconds, the method was sensitive to short term changes in the signal. In the real PWM implementation, activity is classified as healthenhancing only after ten minutes of continuous high activity has been detected, which is consistent with physical activity recommendations [9].

Estimation of energy expenditure from PWM activity data produced reasonably accurate results, although the errors differed rather much between subjects, ranging from 1.2 to $32 \%$. The performance of PWM also varied between tasks. The most accurately estimated tasks were typical everyday tasks, such as walking, vacuuming, and walking up stairs. Energy expenditure was overestimated the most when hands were moving and true energy expenditure was low, e.g., when reading a paper and typing on a computer. The largest underestimations occurred in tasks where true energy expenditure and activity level were high and also when hand movements were absent or limited, e.g., bicycle ergometer and carrying a burden. These results highlight the challenges of wrist based measurement. The heart rate based estimate is less sensitive to movements, and was more accurate in many tasks, especially in high intensity activities.

Our results on estimating energy expenditure with PWM are well in line with the results of previous studies, although most previous studies concerning activity based energy 
expenditure estimation have used uni- or triaxial accelerometers attached at lower back, upper or lower leg, or upper or lower arm. Lower back attachment is near the centre of mass of the body, and has been found the most accurate in energy expenditure estimation [19]. The activity based estimates have been the most accurate in walking tasks and have overestimated low intensity activities and underestimated high intensity activities [19]-[21]. In a study by Bouten et al. [20], a triaxial accelerometer at lower back yielded a $7.5 \%$ overestimation of total energy expenditure in a 36-hour measurement in a respiration chamber, with a set of tasks similar to ours. The wrist attachment has yielded accurate results in walking tasks [19], but poorer results have been achieved when the task set has included also everyday activities. Eston et al. [21] found that $44.2 \%$ of energy expenditure variation was explained by wrist pedometry. In a study by Swartz et al. [22] the result was only $3.3 \%$ for the wrist site. Crouter et al. [23] used a waist-worn accelerometer to classify different types of activities and estimated energy expenditure separately for each class. They obtained a correlation of 0.96 between estimated and true expenditure.

The measurement duration in this study was relatively short because of the strictly controlled procedure as well as the fact that indirect calorimeter reaches steady state rapidly allowing short task durations. On the other hand, indirect calorimeter is uncomfortable in long measurements. A limitation in our study is that the same data were used for developing the algorithms and assessing their performance. To minimize the bias we used leave-one-out cross-validation in the performance estimation. Still, this may cause some positive bias to the results.

\section{CONCLUSION}

The PWM concept was designed to promote personal wellness management by providing information on 24-hour physical activity profile, and especially sleep and healthenhancing physical activity. Continuous, unobtrusive measurement from the wrist allows unique long-term continuous follow-up of activity patterns and sleep. Feedback on the wrist device enables the user to assess his/her behavior related to recommendations of, e.g., health-enhancing physical activity or sleep duration. Wearing the device also reminds the user constantly of the importance of healthy choices. We believe that the concept is suitable for promoting healthy lifestyles and preventing lifestyle related health problems.

\section{REFERENCES}

[1] Y. A. Kesaniemi et al. "Dose-response issues concerning physical activity and health: an evidence-based symposium," Med. Sci. Sports Exerc., 33(6 suppl), pp. S351-8, June 2001.

[2] F. B. Hu, T. Y. Li, G. A. Colditz, W. C. Willett, and J. E. Manson. "Television watching and other sedentary behaviors in relation to risk of obesity and type 2 diabetes mellitus in women," JAMA, vol. 289, pp. 1785-1791, April 2003.

[3] J. L. Hossain and C. M. Shapiro. "The prevalence, cost implications, and management of sleep disorders: An overview," Sleep Breath., vol. 6, pp. 85-102, April 2002.
[4] G. A. Gonzalo and T.A. Najib. "The impact of daily sleep duration on health: A review of the literature,” Prog. Cardiovasc. Nurs, vol. 19, pp. 56-59, February 2004.

[5] N. Cavill, S. Kahlmeier, and F. Racioppi (Eds.). "Physical activity in Europe: evidence for action,” WHO Europe, 2006.

[6] Kansanterveyslaitos, "Elintavat ja niiden väestöryhmäerot Suomessa. Terveys 2000 tutkimus,” Kansanterveyslaitoksen julkaisuja B2/2007, Helsinki, 2007.

[7] Department for Culture, Media and Sport (DCMS) and Strategy Unit. "Game plan: a strategy for delivering Government's sport and physical activity objectives." London, Cabinet Office, 2002.

[8] C. Foster. "Guidelines for health-enhancing physical activity promotion programmes. The European Network for the Promotion of HealthEnhancing Physical Activity," Tampere, the UKK Institute for Health Promotion Research, 2000

[9] W. L. Haskell et al. "Physical activity and public health. Updated recommendation for adults from the American College of Sports Medicine and the American Heart Association," Circulation 2007: published online before print August 2007.

[10] UKK Institute Physical activity pie, available online: www.ukkinstituutti.fi/upload/y8415qkf.pdf

[11] A. Särelä, I. Korhonen, and J. Lötjönen. "IST Vivago® - an intelligent social and remote wellness monitoring system for the elderly", in Proc. 4th Ann. IEEE Conf. Inf. Tech. App. Biomed. Birmingham, UK, pp. 362-365, 2003.

[12] Vivago Personal Wellness Manager, IST International Security Technology [Online]. Available: www.istsec.fi

[13] P. Paavilainen, I. Korhonen, and M. Partinen. 'Telemetric activity monitoring as an indicator of long-term changes in health and well-being of older people," Gerontechnology, vol. 4, pp. 77-85, October 2005.

[14] P. Paavilainen et al. "Circadian activity rhythm in demented and nondemented nursing-home residents measured with telemetric activity", J.Sleep Res., vol. 14, pp. 61-68, March 2005.

[15] J. Lötjönen et al. "Automatic sleep-wake and nap analysis with a new wrist-worn online activity monitoring device IST Vivago WristCare®," Sleep, vol. 26, pp. 86-90, February 2003.

[16] E. Lamminmäki, A. Saarinen, J. Lötjönen, M. Partinen, and I. Korhonen. "Differences in light sleep and deep sleep measured with IST Vivago® WristCare," in Proc. EMBEC'05, pp. 771-775, 2005.

[17] A. S. Jackson et al. "Prediction of functional aerobic capacity without exercise testing," Med. Sci. Sports Exerc., vol. 22, pp. 863-870, July 1990.

[18] Firstbeat Technologies Oy. 2005. VO2 estimation method based on heart rate measurement [Online]. Available: http://www.firstbeattechnologies.com/files/VO2_Estimation.pdf.

[19] C. V. C. Bouten, A. A. H. J. Sauren, M. Verduin, and J.D. Janssen. "Effects of placement and orientation of body-fixed accelerometers on the assessment of energy expenditure during walking," Med. \& Biol. Eng. \& Comput., vol. 35, pp. 50-56, January1997.

[20] C. V. C. Bouten, K. T. M. Koekkoek, M. Verduin, R. Kodde, and J. D. Janssen. "A triaxial accelerometer and portable data processing unit for the assessment of daily physical activity," IEEE Trans. Biomed. Eng, vol. 44, pp. 136-147, March 1997.

[21] R. G. Eston, A.V. Rowlands, and D. K. Ingledew. "Validity of heart rate, pedometry, and accelerometry for predicting the energy cost of children's activities," J Appl. Physiol., 84: 362-371, January 1998.

[22] A. M. Swartz, S. J. Strath, D. R. Basset, W. L. O'Brien, and G. A. King. "Estimation of energy expenditure using CSA accelerometers at hip and wrist sites," Med. Sci. Sports Exerc., vol. 32, pp. S450-S456, September 2000 .

[23] S. E. Crouter, K. G. Clovers, and D. R. Basset, Jr. "A novel method for using accelerometer data to predict energy expenditure," J Appl. Physiol., vol. 100, pp. 1324-1331, April 2006. 